\title{
Myocarditis associated with Plasmodium vivax malaria: a case report
}

\author{
Ana Maria Revoredo da Silva Ventura ${ }^{[1],[2]}$, Tânia do Socorro Souza Chaves ${ }^{[1]}$, \\ Julius Caesar Mendes Monteiro ${ }^{[3]}$, Carina Guilhon Sequeira ${ }^{[2],}$ \\ Maria Deise de Oliveira Ohnishi ${ }^{[2]}$, Rosana Maria Feio Libonati ${ }^{[4]}$, \\ Rita Catarina Medeiros Sousa ${ }^{[4],[5]}$ and Jose Maria de Souza ${ }^{[1]}$
}

[1]. Laboratório de Ensaios Clínicos em Malária, Instituto Evandro Chagas, Secretaria de Vigilância em Saúde, Ministério da Saúde, Belém, PA. [2]. Departamento de Medicina Integrada, Universidade do Estado do Pará, Belém, PA. [3]. Faculdade de Medicina, Universidade Federal do Pará, Belém, PA. [4]. Núcleo de Medicina Tropical, Universidade Federal do Pará, Belém, PA. [5]. Clínica Médica, Hospital Porto Dias, Belém, PA.

\begin{abstract}
Malaria remains a major public health problem in Brazil where Plasmodium vivax is the predominant species, responsible for $82 \%$ of registered cases in 2013. Though benign, P. vivax infection may sometimes evolve with complications and a fatal outcome. Here, we report a severe case of P. vivax malaria in a 35-year-old Brazilian man from a malaria endemic area, who presented with reversible myocarditis.
\end{abstract}

Keywords: Malaria. Reversible myocarditis. Severe Plasmodium vivax malaria.

\section{INTRODUCTION}

Brazil, where malaria is endemic in the Amazon region', is one of the few countries around the world with Plasmodium vivax predominance. We report a case of $P$. vivax malaria in a 35-year-old patient from Anajás, a province in the Island of Marajó, State of Pará $\left(00^{\circ} 98^{\prime} \mathrm{S}, 49^{\circ} 93^{\prime} \mathrm{W}\right)$, which is considered a high-risk area for malaria.

Severe malaria is caused mainly by Plasmodium falciparum. Severe cases similar to those that have been described for $P$. falciparum malaria have been reported with $P$. vivax malaria, but few cases have been reported with cardiac involvement ${ }^{2,3}$.

\section{CASE REPORT}

The 35-year-old male patient was an electrician and 5-year resident of Anajás, State of Pará, Brazil. He had no previous history of malaria or cardiovascular system disorders.

After discharge from the hospital following spinal cord injury due to an occupational accident, the patient presented with a 5-day history of fever, chills, headache, and asthenia,

Address to: Dra. Ana Maria Revoredo da Silva Ventura. Laboratório de Ensaios Clínicos em Malária/IEC. Rodovia BR-316, km 7 s/n, Levilândia, 67030-000 Ananindeua, PA, Brasil.

Phone: 5591 3226-5880; 5591 8146-7723

e-mail: ana_mariaventura@hotmail.com

Received 30 May 2014

Accepted 21 August 2014 and was again hospitalized. In the hospital, a thick blood smear was positive to $P$. vivax $\left(30,000\right.$ asexual parasite forms $\left./ \mathrm{mm}^{3}\right)$, and he was treated with $150 \mathrm{mg}$ chloroquine (4 tablets on the first day and 3 tablets on the second and third days) plus $15 \mathrm{mg}$ primaquine ( 2 tablets/day for 7 days) according to the treatment regime proposed by the Health Ministry of Brazil ${ }^{4}$.

In the third day of hospitalization, primaquine was discontinued and the patient transferred to the intensive care unit due to worsening respiratory distress concomitant with signs of heart failure and petechial hemorrhagic suffusion on the thorax, abdomen and lower limbs (Figure 1). Normal white blood cell count observed at admission evolved to leukopenia $\left(3,000 / \mu \mathrm{L} / \mathrm{mm}^{3}\right)$, and hemoglobin decreased from $12.8 \mathrm{~g} / \mathrm{dL}$ to $8.9 \mathrm{~g} / \mathrm{dL}$. Thrombocytopenia was present both at admission $\left(20,000\right.$ platelets $\left./ \mathrm{mm}^{3}\right)$ and in day $3\left(15,000\right.$ platelets $\left./ \mathrm{mm}^{3}\right)$. Chest radiography revealed pulmonary edema and cardiomegaly (Figures 2A and 2B), Ecocardiography revealed left ventricular dilatation during systole $(5 \mathrm{~mm}$, normal $=4 \mathrm{~mm})$ and diastole $(73 \mathrm{~mm}$, normal $=56 \mathrm{~mm})$, decreased left ventricular ejection fraction (LVEF) (47\%, normal > 58\%), diffuse hypokinesia and mild mitral regurgitation (Figure 3). Blood and urine cultures were negative. Results of serological tests for dengue, yellow fever, infectious mononucleosis, Chagas disease, enterovirus (Coxsackie and Echovirus), human immunodeficiency virus (HIV) and human T-lymphotropic virus (HTLV) were also negative. Polymerase chain reaction (PCR) to confirm $P$. vivax infection was not performed.

The patient gradually improved with antibiotic therapy (oxacillin and ceftriaxone), administered with antimalarials due to the clinical severe condition. He was also administered diuretics (furosemide and spironolactone), digoxin and 


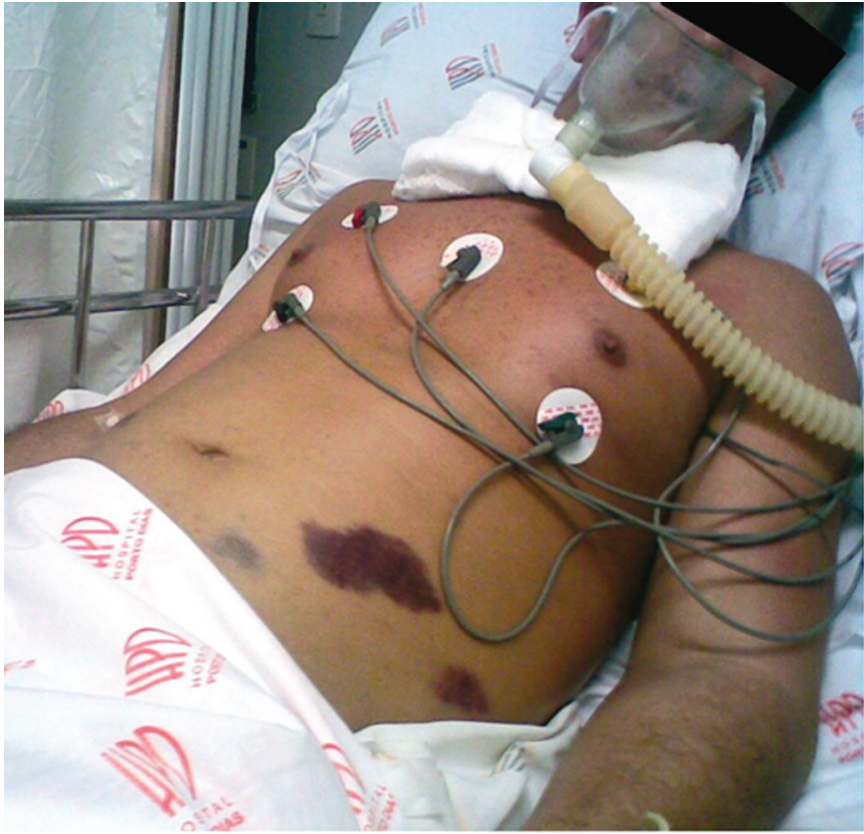

FIGURE 1 - Petechial hemorrhagic suffusion, on the thorax and abdomen of a patient with Plasmodium vivax malaria.

carvedilol and was maintained in negative fluid balance. A thick blood smear was negative to Plasmodium sp to day 5, and after 12 days of treatment, he was discharged (February, $11^{\text {th }}$, 2009 ) in regular health and administered carvedilol (1 tablet, 2 times/day).

Thereafter, he did not return to Anajás and was monitored on a monthly basis in the Laboratory of Clinical Malaria Essay at the Evandro Chagas Institute, in Ananindeua, State of Pará, Brazil, relapsing on the $46^{\text {th }}$ day post-treatment, with 3,500 asexual $P$. vivax parasite forms $/ \mathrm{mm}^{3}$. At this time, the standard treatment was reintroduced. A negative blood smear was observed on day 4, which persisted for up to 90 days with monthly controls. In May - 2009, radiography showed normal lung transparency and normal cardiac area; electrocardiography showed normal sinus rhythm; and echocardiography showed a 34-mm ventricular diameter in systole, 50 -mm left ventricular diameter in diastole, LVEF of $60 \%$, and heart valves without morphologic or dynamic abnormalities.

\section{DISCUSSION}

The worldwide prevalence of severe $P$. vivax malaria cases is not well known, probably because there are no defined severity criteria for malaria caused by P. vivax. However, the World Health Organization (WHO) criteria for severe $P$. falciparum malaria seem to apply to the broad spectrum of the most severe P.vivax malaria cases described ${ }^{3,5}$.

A Plasmodium vivax malaria case complicated with myocardial failure and hemorrhagic diathesis in an adult man from a malaria endemic area (Anajás) in the Amazon region (Brazil) without a previous history of malaria, was reported
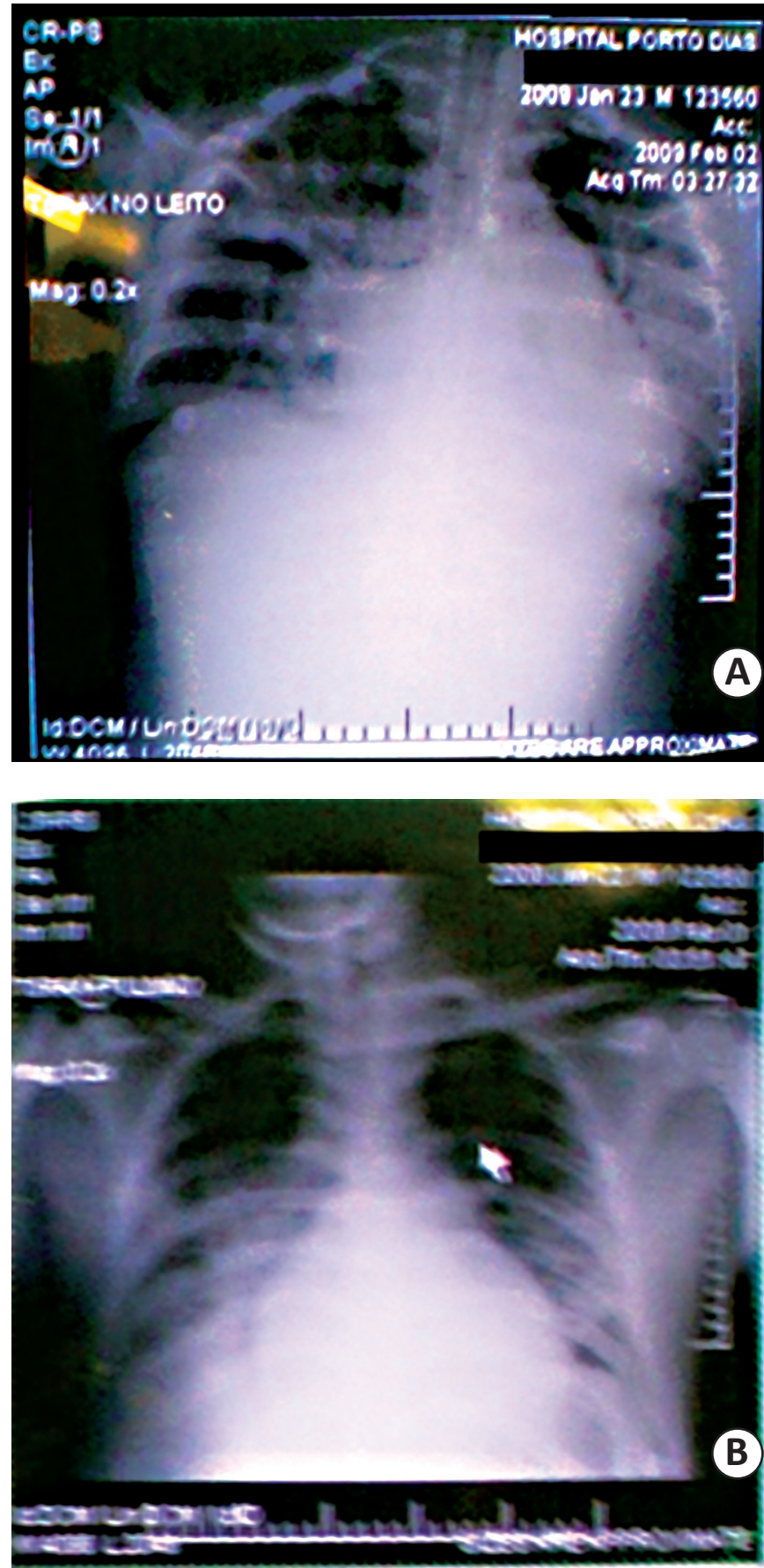

FIGURES 2A and 2B - Chest radiography showing cardiomegaly and acute edema of the lung in a patient with Plasmodium vivax malaria.

in addition to the first $P$. vivax malaria episode in six patients who presented with cardiac involvement (from a series of 22 malaria cases admitted in a German hospital) ${ }^{2}$. Similarly a case of myocarditis associated with primary $P$. vivax malaria has been reported in a woman from South Korea ${ }^{3}$. Though these primary cases were related to the absence of specific immunity to the parasite, severe cases, including cardiac damage, may occur in patients with previous malaria who have some degree 

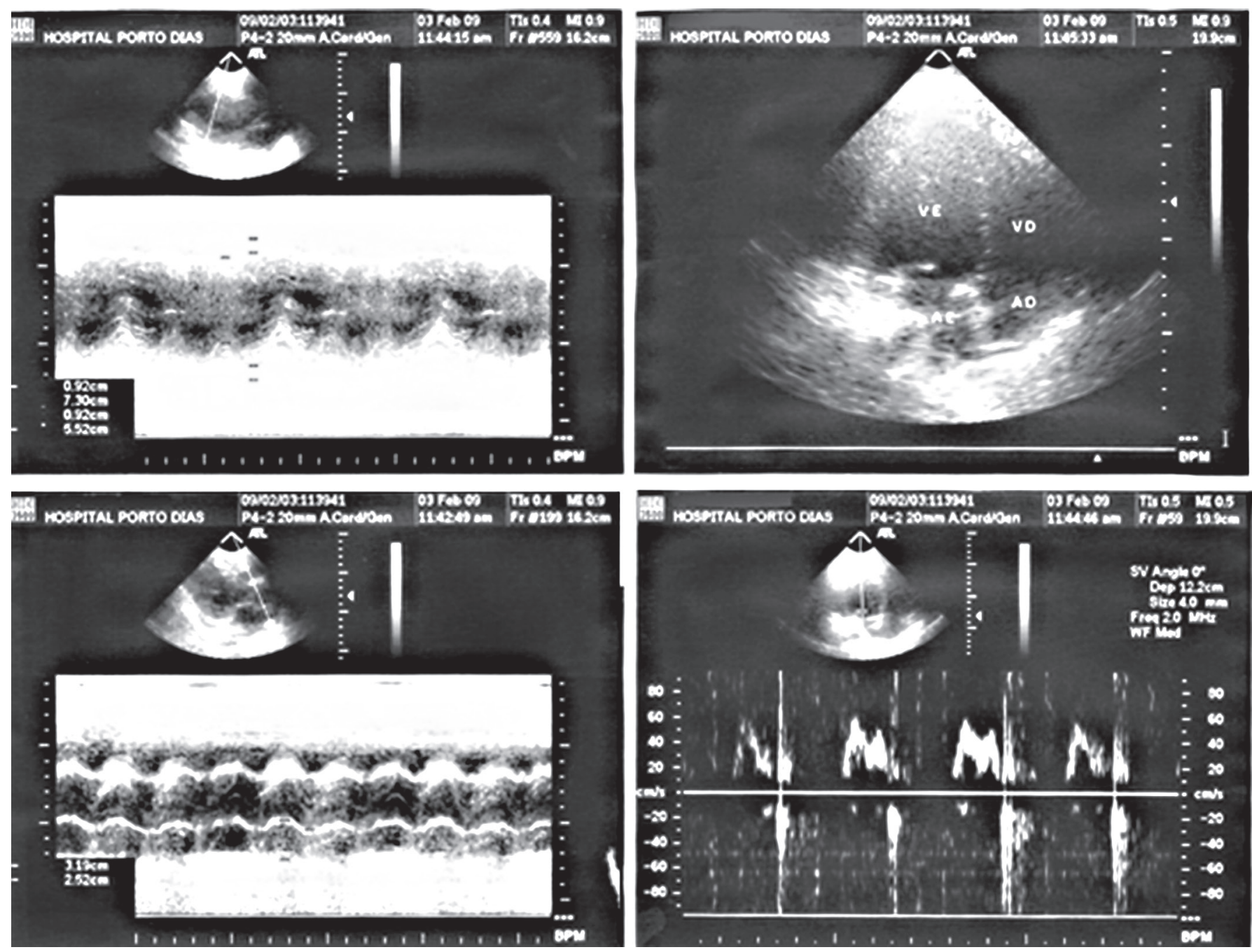

FIGURE 3 - Echocardiography showing left ventricular dilatation in systole and diastole and mild mitral regurgitation in a patient with Plasmodium vivax malaria.

of immunity, making this relationship between myocarditis and malaria unclear ${ }^{2}$.

Our patient presented with high parasitemia (30,000 asexual parasite forms $/ \mathrm{mm}^{3}$ ), which might have influenced the severity, although additional studies are necessary to determine to what extent high parasite density may be considered a marker of severity in $P$. vivax infections, as indicated by Lacerda et al. ${ }^{6}$.

In a recent review of cardiac involvement in parasitic infection, Hidron et al. reported that Trypanosoma cruzi is the main parasite to compromise the heart; however the authors did not mention malaria ${ }^{7}$. Myocardial involvement in malaria seems uncommon, with unclear physiopathology in the majority of cases associated with P. falciparum. Autopsy findings have shown parasites and parasitized red blood cells blocking myocardial capillaries, leading to ischemic cardiomyopathy, and a dilated heart has also been observed. In addition, the toxic effects of high levels of tumor necrosis factor (TNF) may play a role in the inflammatory process in the heart, with migration of lymphocytes and plasma cells, among others ${ }^{3,8}$.
Besides cardiac involvement, the present patient manifested signs of septic shock. Myocardial dysfunction is one of the diagnostic criteria for severe sepsis and septic shock and frequently accompanies these conditions, presenting as ventricular dilatation and reduced ejection fraction; when properly managed, it is reversible. An infection stimulus, generally an endotoxin or another microbiological element (e.g., malaria endotoxins), - induces the release of local and systemic inflammatory mediators, especially alpha-TNF and interleukin (IL)-1 $\beta$, from monocytes/macrophages and other cells. These cytokines stimulate polymorphonuclear leukocytes, macrophages, and endothelial cells to release a number of downstream inflammatory mediators, including platelet activating factor and nitric oxide, further amplifying the inflammatory response. Several anti-inflammatory mediators, such as IL-10, transforming growth factor-beta, and IL-1 receptor antagonist, are also released as part of this amplification cascade?

The myocardial involvement in the present patient was probably caused by the parasite itself and/or due to an inflammatory-trigged response; the physiopathology of the events 
that occur in $P$. vivax and $P$. falciparum infections reflects the immune response (mainly an unbalance of pro and anti-inflammatory cytokines) of the host towards the parasite, which isusually more prominent in the first Plasmodium infection ${ }^{10}$, as was observed in this case. Other infections responsible for compromised myocardial function were ruled out: blood and urine cultures, dengue, yellow fever, infectious mononucleosis, Chagas disease, enterovirus (Coxsackie and Echovírus), HIV and HTLV serology were negative.

Though chloroquine can cause cardiomyopathy, particularly with long-term use ${ }^{11}$, the clinical manifestation of heart involvement, including tachycardia, dyspnea, and fatigue with minimal exertion, in this patient were present before $P$. vivax diagnosis and administration of chloroquine.

Despite a lack of $P$. vivax confirmation or P. falciparum exclusion by PCR, the following three aspects of the present case strongly suggest $P$. vivax etiology: a) several thick blood slides were positive to $P$. vivax during hospitalization, determined by an expert microscopist, from a reference Malaria Diagnosis Center, and there was a prompt response to chloroquine with a decrease in and clearance of parasitemia; $b$ ) in Brazil, strains of $P$. falciparum are usually resistant to chloroquine ${ }^{12}$; and c) the relapse during the follow up, which was expected to a certain extent because primaquine was discontinued due patient's worse clinical condition, included lung involvement, heart failure, petechial hemorrhagic suffusion, and thrombocytopenia.

This case report demonstrates that $P$. vivax infection can evolve with severe complications and poor outcomes. Though uncommon, myocarditis can occur with $P$. vivax malaria and health professionals should be aware of this possibility for appropriate monitoring and management.

\section{REFERENCES}

1. Costa FTM, Lopes SCP, Albrecht L, Ataíde R, Siqueira AM, Souza $\mathrm{RM}$, et al. On the pathogenesis of Plasmodium vivax malaria:
Perspectives from the Brazilian field. Int J Parasitol 2012; 42: 1099-1105.

2. Franzen D, Curtius JM, Heitz W, Hopp HW, Diehl V, Hilger HH. Cardiac involvement during and after malaria. Clin Investig 1992; 70:670-673.

3. Kim SA, Kim ES, Rhee MY, Choi SI, Huh HJ. A case of myocarditis associated with Plasmodium vivax. J Travel Med 2009; 16: 138-140.

4. Ministério da Saúde do Brasil. Secretaria de Vigilância em Saúde. Departamento de Vigilância Epidemiológica. Guia prático de tratamento da malária no Brasil. Série A. Normas e Manuais Técnicos. Brasília: Ministério da Saúde; 2010.

5. World Health Organization. Severe falciparum malaria. Trans R Soc Trop Med Hyg 2000; 94 (supl 1):S1-S90.

6. Lacerda MVG, Mourão MPG, Alexandre MAA, Siqueira AM, Magalhães BML, Martinez-Espinosa FE, et al. Understanding the clinical spectrum of complicated Plasmodium vivax malaria: a systematic review on the contributions of the Brazilian literature. Malar J 2012; 11:12

7. Hidron A, Vogenthaler N, Santos-Preciado JI, Rodriguez-Morales AJ, Franco-Paredes C, Rassi Jr A. Cardiac involvement with parasitic infections. Clin Microbiol Rev 2010; 324-349.

8. Gupta N, Sahoo SK. Plasmodium vivax induced myocarditis: A rare case report. Indian J Med Microbiol 2013; 31:180-181.

9. Tal S, Guller V, Goland S, Shimoni S, Gurevich A. Reversible myocardial dysfunction in septic shock. Isr Med Assoc J 2013; 15:588-589.

10. Mendonça VR, Queiroz AT, Lopes FM, Andrade BB, Barral-Netto M. Networking the host immune response in Plasmodium vivax malaria. Malar J 2013; 12:69.

11. Tönnesmann E, Kandolf R, Lewalter T. Choloroquine cardiomiopathy- a review of the literature. Immunopharmacol Immunotoxicol 2013; 35:434-442.

12. Vieira PP, Ferreira MU, Alecrim MG, Alecrim WD, da Silva LH, Sihuincha MM, et al. pfcrt Polymorphism and the spread of chloroquine resistance in Plasmodium falciparum populations across the Amazon Basin. J Infect 2004; 190:417-424. 